\title{
Aerobic Exercise During Encoding Impairs Hippocampus-Dependent Memory
}

\author{
Keishi Soga, Keita Kamijo, and Hiroaki Masaki \\ Waseda University
}

\begin{abstract}
We investigated how aerobic exercise during encoding affects hippocampus-dependent memory through a source memory task that assessed hippocampus-independent familiarity and hippocampus-dependent recollection processes. Using a within-participants design, young adult participants performed a memory-encoding task while performing a cycling exercise or being seated. The subsequent retrieval phase was conducted while sitting on a chair. We assessed behavioral and event-related brain potential measures of familiarity and recollection processes during the retrieval phase. Results indicated that source accuracy was lower for encoding with exercise than for encoding in the resting condition. Event-related brain potential measures indicated that the parietal old/new effect, which has been linked to recollection processing, was observed in the exercise condition, whereas it was absent in the rest condition, which is indicative of exercise-induced hippocampal activation. These findings suggest that aerobic exercise during encoding impairs hippocampusdependent memory, which may be attributed to inefficient source encoding during aerobic exercise.
\end{abstract}

Keywords: event-related potentials, familiarity, recollection, source memory

Increasing empirical evidence suggests that physical activity or physical fitness across the human life span is associated with hippocampal structure and function (Chaddock et al., 2010; Chaddock, Hillman, Buck, \& Cohen, 2011; Erickson et al., 2009, 2011; see also Erickson, Hillman, \& Kramer, 2015 for a review). For example, a cross-sectional study using a hippocampusdependent relational memory task in children (Chaddock et al., 2010) has indicated that greater aerobic fitness is associated with larger hippocampal volume and superior memory performance. A longitudinal, randomized controlled intervention study has also indicated that a 1-year aerobic fitness training program increased hippocampal volume in older adults (Erickson et al., 2011). These findings imply that regular physical activity is related to hippocampal development and health.

From a different perspective, several studies have explored the effects of a single bout of acute exercise on memory functions. These studies have consistently indicated that retrieval performance improved when learning or when the encoding phase was performed after acute aerobic exercise (Etnier, Labban, Piepmeier, Davis, \& Henning, 2014; Labban \& Etnier, 2011; Pesce, Crova, Cereatti, Casella, \& Bellucci, 2009; Salas, Minakata, \& Kelemen, 2011; Winter et al.,

Keishi Soga is with the Graduate School of Sport Sciences, Waseda University, Tokorozawa, Japan. Keita Kamijo and Hiroaki Masaki are with the Faculty of Sport Sciences, Waseda University, Tokorozawa, Japan. Address author correspondence to Hiroaki Masaki at masaki@ waseda.jp.
2007), suggesting the beneficial effects of acute exercise on hippocampal functioning. The positive effects of acute exercise on memory have been validated by several meta-analyses (Chang, Labban, Gapin, \& Etnier, 2012; Lambourne \& Tomporowski, 2010; Roig, Nordbrandt, Geertsen, \& Nielsen, 2013). Based on these findings, it has been proposed that, for example, physically active academic lessons, in which exercise and learning activities are simultaneously conducted (Donnelly \& Lambourne, 2011; Mullender-Wijnsma et al., 2015), might increase both physical activity level and learning efficiency. However, several studies have found a deterioration in executive function (i.e., higher order cognitive abilities involved in goal-directed behaviors) during aerobic exercise (Pontifex \& Hillman, 2007; Soga, Shishido, \& Nagatomi, 2015). Thus, the effects of acute exercise on brain function are likely to differ depending on the timing of administering the cognitive task (i.e., after or during exercise). A metaanalysis conducted by Lambourne and Tomporowski (2010) support this view by suggesting that cognitive task performance improves after exercise but deteriorates during exercise. Another meta-analysis has indicated that acute exercise, in general, has positive effects on cognitive functions (Chang et al., 2012). However, the effects of simultaneous exercise on cognitive functions appear to differ depending on the duration of exercise. When cognitive tasks were performed during exercise, exercise had adverse effects for 11-20 min and benefits after $20 \mathrm{~min}$. Although these recent metaanalyses have indicated the effects of simultaneous 
exercise on cognitive function, studies on the effects of acute exercise during the encoding stage on memory functions have been infrequent and controversial; moreover, the existing evidence shows both negative (Miles \& Hardman, 1998) and positive effects (SchmidtKassow et al., 2013, 2014). Hence, this study was designed to clarify how hippocampus-dependent memory functions are affected when the encoding phase of a memory task is conducted during acute aerobic exercise.

Miles and Hardman (1998) examined the effects of aerobic exercise during encoding on retrieval performance, by focusing on state-dependent learning, in which participants show better retrieval performance when they are in the same state as during the encoding phase. Specifically, encoding (word list learning) and retrieval (free recall) phases were performed during both seated rest and moderate aerobic exercise (i.e., in within-participants $2 \times 2=4$ conditions design: rest-rest, rest-exercise, exercise-rest, and exercise-exercise for encoding-retrieval, respectively). Results indicated that participants exhibited better free recall performance in the same-state encoding-retrieval condition (i.e., restrest and exercise-exercise) relative to changed-state encoding-retrieval condition (i.e., rest-exercise and exercise-rest), supporting the state-dependent learning account. That is, focusing on the case in which the retrieval phase was performed during seated rest (i.e., comparison between rest-rest and exercise-rest conditions) indicated that simultaneous exercise while encoding deteriorated retrieval performance.

In contrast to studies suggesting negative effects of exercise during the encoding stage on retrieval performance during the resting state, Schmidt-Kassow et al. (2013, 2014) indicated that aerobic exercise during encoding improved vocabulary learning. Specifically, in a study using a between-participants design, native German participants learned Polish vocabulary during seated rest or moderate-intensity cycling exercise, and then performed the retrieval phase during seated rest. Results indicated that participants in the exercise group exhibited better retrieval performance relative to the seated rest group (Schmidt-Kassow et al., 2013). Schmidt-Kassow et al. (2014) extended this finding using the identical learning task and reported that walking (i.e., low-intensity aerobic exercise) during vocabulary encoding improved retrieval performance. In summary, research on the effects of aerobic exercise during encoding on hippocampus-dependent memory functioning remains contradictory.

To clarify this issue, we employed a source memory task (Figure 1a and 1b) that could manipulate hippocampal neuronal functioning based on task conditions. The source memory task asked participants to make a size judgment (smaller or bigger than a shoe box) or an animacy judgment (living or nonliving) and to encode those items during the encoding phase. In the subsequent retrieval phase, participants were required to make an old or new judgment, and if old, to make a source judgment (i.e., whether the item was seen in the size block or the animacy block). The dual-process model postulates two types of memory retrieval processes: familiarity and recollection. Familiarity refers to the feeling that an item or event has occurred in the past without any concurrent contextual information, whereas recollection refers to the memory for an item or event that is accompanied by the retrieval of contextual aspects of the encoding episode (Cabeza, Ciaramelli, Olson, \& Moscovitch, 2008; Dolcos, LaBar, \& Cabeza, 2005). These two processes involve different neural networks (Yonelinas, Aly, Wang, \& Koen, 2010). Therefore, the accuracy of old or new judgments and source accuracy of the source memory task can be used as measures of familiarity and recollection, respectively. Neuroimaging studies have indicated that hippocampus plays a critical role in the recollection process, but not in the familiarity process (Cansino, Maquet, Dolan, \& Rugg, 2002; Hoppstadter, Baeuchl, Diener, Flor, \& Meyer, 2015; Yonelinas, Otten, Shaw, \& Rugg, 2005; see also Eichenbaum, Yonelinas, \& Ranganath, 2007; Konkel \& Cohen, 2009 for reviews).

Prior studies have indicated that the effects of acute aerobic exercise are disproportionately larger for task conditions requiring increased executive function (Dietrich \& Sparling, 2004; Hillman et al., 2009; Pontifex \& Hillman, 2007; Pontifex, Hillman, Fernhall, Thompson, \& Valentini, 2009; Weng, Pierce, Darling, \& Voss, 2015). For example, Pontifex and Hillman (2007) used a modified flanker task and demonstrated that deterioration in task performance during aerobic exercise was selectively observed for incongruent trials necessitating greater demands on executive function, but not for congruent trials that made minimal demands. Moreover, it is well known that the dorsolateral prefrontal cortex (DLPFC) plays a crucial role in the regulation of executive function (Alvarez \& Emory, 2006; Funahashi, 2001; Smith \& Jonides, 1999). Thus, it is possible that the effects of acute aerobic exercise are disproportionately greater for cognitive processes that are mediated by the DLPFC. Neuroimaging studies have demonstrated that DLPFC activity was greater during associative compared with item encoding and that DLPFC activation predicted subsequent associative memory performance but not item memory performance (Murray \& Ranganath, 2007), suggesting that the DLPFC also plays a major role in encoding context information. Therefore, we assumed that the effects of aerobic exercise would be disproportionately greater for the recollection process, which requires both hippocampal and DLPFC functions, than for the familiarity process.

The source memory task allows for assessing familiarity and recollection processes using event-related brain potentials (ERPs) by taking advantage of their high temporal resolution. ERP studies have indicated that correctly remembered old items elicit more positivegoing waveforms than correctly rejected new items (Curran, 2000; Curran \& Hancock, 2007; Herzmann, Jin, Cordes, \& Curran, 2012; Senkfor \& Van Petten, 1998; Tsivilis et al., 2015; Wilding \& Rugg, 1996; Woroch \& Gonsalves, 2010). This so-called old/new 
(a)

$$
\text { Encoding phase }
$$

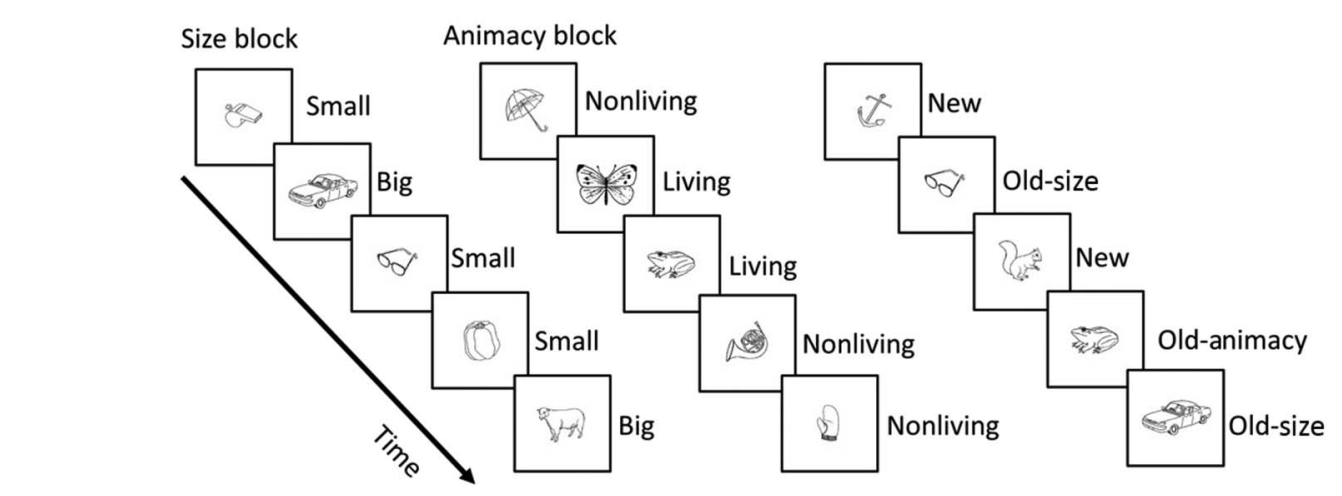

Retrieval phase

(b)

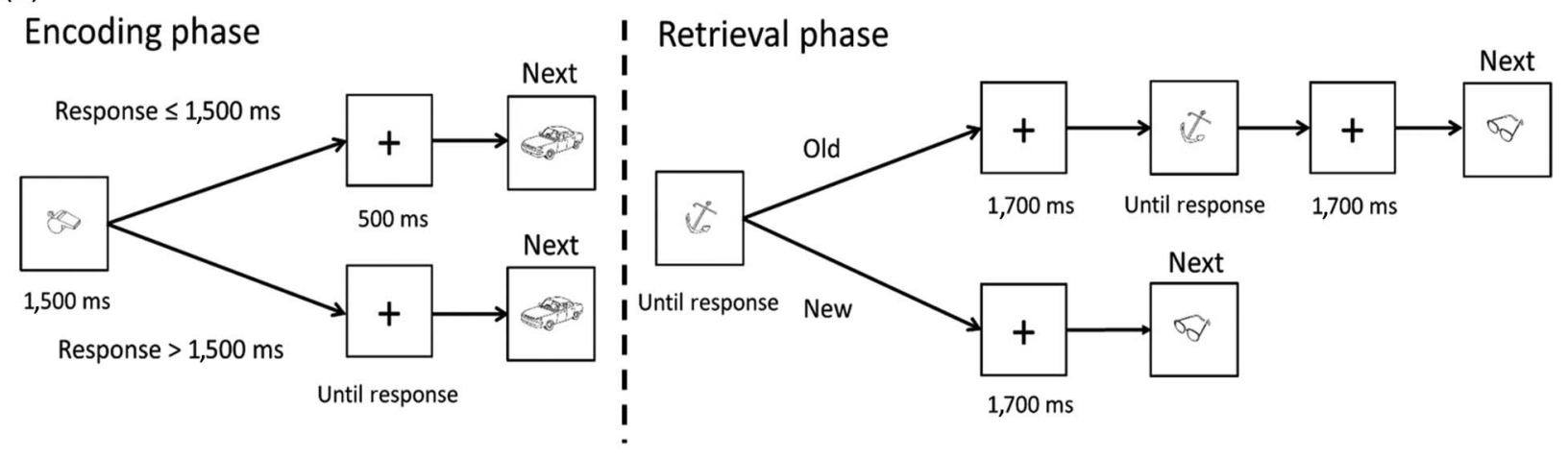

(c)
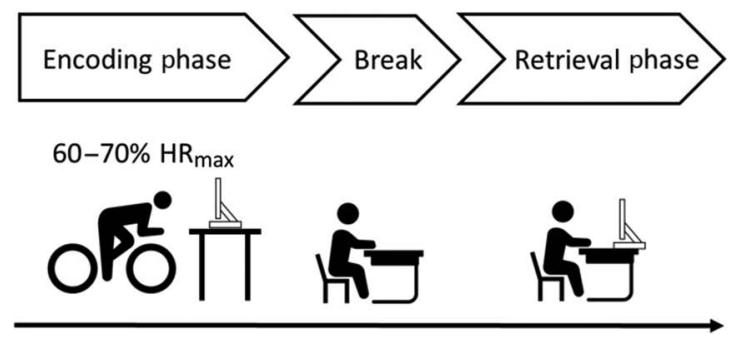
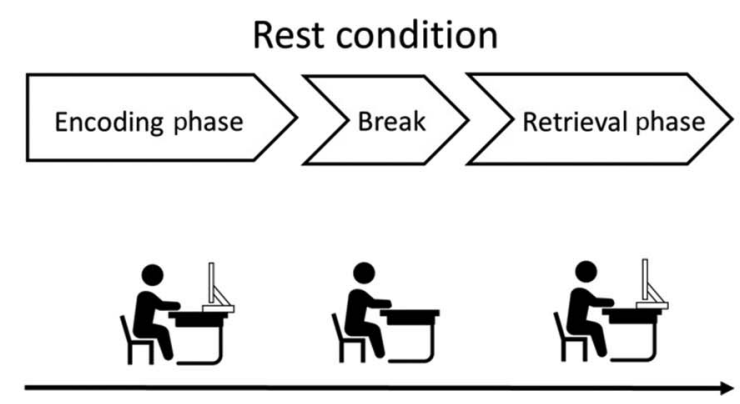

Figure 1 - (a) Examples of stimuli and correct judgments in the encoding and retrieval phase. (b) Schematic illustrations of the sequence of events in the encoding and retrieval phase. (c) Experimental protocol for exercise and control conditions.

effect can be separated into two temporally and topographically distinct components: the frontal N400-like component (FN400) old/new effect and parietal old/new effect, occurring roughly $200-400 \mathrm{~ms}$ and 400-900 ms following stimulus onset, respectively. Previous studies using source memory tasks compared ERP amplitudes on both correct and incorrect source trials with ERP amplitudes on correct rejection trials to assess the old/ new effects. These studies have shown that both correct source and incorrect source trials exhibited the FN400 old/new effect (i.e., correct source $>$ correct rejection, incorrect source $>$ correct rejection), whereas only correct source trials, but not incorrect source trials, exhibited the parietal old/new effect (i.e., correct source $>$ correct rejection, incorrect source $\approx$ correct rejection;
Addante, Ranganath, \& Yonelinas, 2012; Cansino \& Trejo-Morales, 2008; Mollison \& Curran, 2012; Peters \& Daum, 2009). ${ }^{1}$ Based on these findings, it has been proposed that the retrieval processes of familiarity and recollection are associated with the FN400 old/new effect and parietal old/new effect, respectively (Addante et al., 2012; Curran \& Cleary, 2003; Wilding, 2000; Wilding \& Rugg, 1996; Woroch \& Gonsalves, 2010). Wilding (2000) further indicated that an increased number of correct source judgments were associated with increased parietal old/new effect. In addition, a neuroimaging study has suggested that the parietal old/new effect reflects hippocampal activation (Hoppstadter et al., 2015). Collectively, the parietal old/new effect is thought to reflect brain activity involved in the source 
retrieval process (i.e., recollection) and negative and positive effects of aerobic exercise on the recollection processes would be denoted by a decreased or increased parietal old/new effect, respectively.

The present study investigated the effects of acute aerobic exercise during encoding on hippocampusdependent memory function using a source memory task. We expected that the effects of aerobic exercise would be selectively and disproportionately greater for the hippocampus-dependent recollection process, which would be reflected by changes in source accuracy and the parietal old/new effect. In contrast, we predicted that old or new accuracy and the FN400 old/new effect reflecting the familiarity process would be less influenced by acute aerobic exercise. Prior studies have provided conflicting findings regarding the direction of these effects (i.e., negative or positive) of acute aerobic exercise on retrieval performance (Miles \& Hardman, 1998; SchmidtKassow et al., 2013, 2014). Accordingly, although we expected that negative and positive effects of aerobic exercise would be reflected by decreased and increased source accuracy and parietal old/new effect, respectively, we did not make any specific a priori predictions about the direction of the effects.

\section{Methods}

\section{Participants}

Twenty undergraduate and graduate students were recruited from Waseda University. All participants were free of neurological diseases or physical disabilities. Data from two participants were discarded because of insufficient trials for averaging ERP $(<10$ trials; Addante et al., 2012; Mollison \& Curran, 2012; Woroch \& Gonsalves, 2010). Thus, we analyzed behavioral and ERP data of 18 participants (5 women: mean age $=$ 22.3 years, $S D=2.4$ ). Table 1 illustrates participants' demographic information. All participants provided written informed consent before participating in the study. The ethics committee on human research of Waseda University approved the protocol of this study.

\section{Procedure}

To eliminate individual differences, we employed a within-participants design, in which participants performed the encoding phase during aerobic exercise and during seated rest on two separate days. Participants were counterbalanced across conditions to minimize practice effects, such that half performed the exercise condition first and the other half performed the rest condition first. On the first day, participants completed questionnaires including the Physical Activity Readiness Questionnaire to screen those with potential medical risks that could be exacerbated by acute exercise (Thomas, Reading, \& Shephard, 1992) and the Global Physical Activity Questionnaire to assess the amount of physical activity in a typical week (metabolic equivalentmin/week; Bull, Maslin, \& Armstrong, 2009). Participants' height and weight were measured using a Tanita WB-300 digital scale (Tanita Corp., Tokyo, Japan).

Before the experiment began, participants were fitted with a 64-channel head cap with $\mathrm{Ag} / \mathrm{AgCl}$ active electrodes (ActiveTwo system; Biosemi, Amsterdam, The Netherlands) and a polar heart rate (HR) monitor (RS400; Polar, Kempele, Finland). Participants then engaged in a short practice block of about $5 \mathrm{~min}$. The HR was recorded throughout the test. Figure 1c illustrates the experimental protocol for both exercise and control conditions. In the exercise condition, participants sat on a Monark 828E cycle ergometer (Monark, Vansbro, Sweden) for $2 \mathrm{~min}$ and then pedaled at 60 revolutions per minute for $5 \mathrm{~min}$ for warming-up, during which the workload was gradually increased to reach the target HR (60-70\% estimated maximum HR: $\mathrm{HR}_{\max }$, 208-0.7 ×age; Tanaka, Monahan, \& Seals, 2001). A meta-analysis has suggested that exercise intensity has a nonsignificant effect on cognitive function when cognitive tasks are performed during exercise (Chang et al., 2012). Thus, the moderate intensity was chosen based on previous studies that have investigated the effects of simultaneous exercise on cognitive functions. As indicated in the beginning of this article, because the effects of simultaneous exercise on cognitive functions are still controversial, we did not make any specific a priori predictions concerning the direction of the effects (i.e., negative or positive). To clarify the discrepancy among these studies, we relied on previous studies that have indicated both negative (Pontifex \& Hillman, 2007; Soga et al., 2015) and positive effects (Schmidt-Kassow et al., 2013). After participants reached the target HR, the workload was controlled by the experimenter, such as to maintain the target HR (mean HR during

Table 1 Mean (SD) Values for Participant Demographic Data

\begin{tabular}{lccc}
\hline Variable & All & Males & Females \\
\hline No. of participants & 18 & 13 & 5 \\
Age (years) & $22.3(2.4)$ & $22.8(2.7)$ & $21.2(1.1)$ \\
Height $(\mathrm{cm})$ & $168.2(7.2)$ & $171.8(4.6)$ & $158.9(3.2)$ \\
Weight $(\mathrm{kg})$ & $63.5(9.3)$ & $66.9(7.8)$ & $54.7(7.2)$ \\
Body mass index $\left(\mathrm{kg} / \mathrm{m}^{2}\right)$ & $22.4(2.9)$ & $22.7(3.0)$ & $21.7(2.7)$ \\
GPAQ (metabolic equivalent-min/week) & $3595.6(3002.8)$ & $2935.4(2023.1)$ & $5312.0(4576.3)$ \\
\hline
\end{tabular}

Note. GPAQ = Global Physical Activity Questionnaire. 
exercise $=128.0$ beats per minute, $S D=5.9$ ). After the warming-up period, participants started performing the encoding phase. We instructed participants to continue pedaling at 60 revolutions per minute until the end of the encoding phase. They were then given a 5-min break. Finally, the retrieval phase was conducted while participants sat on a chair. In the rest condition, participants performed the same procedure as in the exercise condition, but both the encoding and retrieval phases were done while sitting on a chair. The mean durations of the encoding and retrieval phase across conditions were $7.0 \mathrm{~min}(S D=0.4 \mathrm{~min})$ and $19.8 \mathrm{~min}(S D=2.4 \mathrm{~min})$, respectively.

\section{Stimuli}

A total of 504 black and white outline pictures were selected from the following studies: Bonin, Peereman, Malardier, Meot, and Chalard (2003); Cycowicz, Friedman, Rothstein, and Snodgrass (1997); Nishimoto, Ueda, Miyawaki, Une, and Takahashi (2012); and external sources. All pictures were assigned to four categories: small/living, small/nonliving, big/living, and big/ nonliving. Twenty-four pictures were used for the practice block. Twenty pictures from each category were randomly selected from the remaining 480 pictures to create six sets of 80 pictures (three sets for the exercise condition and the other three sets for the rest condition). For each exercise and rest condition, two sets of 80 pictures (one set for the size judgment block and another set for the animacy judgment block) were used for the encoding phase. During the retrieval phase, the studied 160 pictures and 80 new pictures were presented. All pictures were presented at the center of a display with a resolution of $400 \times 400$ pixels using Presentation (Neurobehavioral Systems, Inc., Berkeley, CA). Each picture was a different size and subtended from $2^{\circ}$ to $9^{\circ}$ of visual angle. The viewing distance was approximately $60 \mathrm{~cm}$.

\section{Task}

Figure 1a and 1b show examples of stimuli and schematic illustrations of the sequence of events in the encoding and retrieval phases. In the encoding phase, participants performed a size judgment block and an animacy judgment block, and they were instructed to memorize the presented pictures. In the size block, participants were asked to press one of two buttons with their thumb corresponding to whether a presented picture was smaller or larger than a standard shoe box, whereas in the animacy block, they were asked to judge whether a presented picture was living or nonliving. After a fixation cross was displayed for $500 \mathrm{~ms}$, a picture was presented on a white screen for 1,500 ms. When participants responded within $1,500 \mathrm{~ms}$, a fixation cross was presented immediately after the stimulus offset for $500 \mathrm{~ms}$, and the next picture was presented. When their reaction times (RTs) were longer than 1,500 $\mathrm{ms}$, the fixation cross was presented until a response was made and the next picture was presented $500 \mathrm{~ms}$ after the response.

In the retrieval phase, participants were asked to press one of two buttons corresponding to whether a presented picture was old or new, and if old, they were required to press one of two buttons corresponding to whether the picture was seen in the size block or in the animacy block. All pictures were displayed until participants responded. When participants judged a picture as old, irrespective of response correctness, a fixation was presented immediately after their response for $1,700 \mathrm{~ms}$, and then the same picture was presented again until they made the source judgment. After a 1,700-ms fixation display, the next picture was presented. When participants judged a picture as new, a fixation was presented after their response for $1,700 \mathrm{~ms}$, and then the next picture was presented. The order of the size block and animacy block, and the stimulus-response mapping were counterbalanced across participants and conditions.

Responses were classified into six categories: (a) hit was defined as the correct identification of old pictures as old, (b) miss was defined as the incorrect identification of old pictures as new, (c) correct source was defined as the correct identification of source information, (d) incorrect source was defined as the incorrect identification of source information, (e) correct rejection was defined as the correct identification of new pictures as new, and (f) false alarm was defined as the incorrect identification of new pictures as old. We used hit, correct source, and correct rejection accuracy for the analysis. Trials with RTs longer than $2 S D$ from each participant's mean were excluded from the analysis. For initial old/new responses, trials with RTs shorter than $200 \mathrm{~ms}$ were also excluded from the analysis.

\section{ERP Recording}

Electroencephalography (EEG) data obtained during the retrieval phase are reported, whereas those taken during the encoding phase are not reported because the analysis of EEG data during the encoding phase in the exercise condition was technically difficult due to excessive noise (i.e., drifts caused by sweat and muscle activity) in EEG data. EEG activity was recorded from 64 electrode sites arranged in an extended montage, based on the international 10-10 system (Chatrian, Lettich, \& Nelson, 1985). Additional electrodes were placed above and below the left orbit and on the outer canthus of both eyes to monitor electro-oculographic activity with a bipolar recording. Continuous data were digitized at a sampling rate of $1024 \mathrm{~Hz}$ with a bandwidth of DC to $205 \mathrm{~Hz}$ ( $-3 \mathrm{~dB} /$ octave), using the Biosemi ActiveTwo system. We analyzed offline EEG data using Brain Vision Analyzer 2 software (Brain Products, Gilching, Germany). The analysis included re-referencing to average mastoids, band-pass filtering $(0.1-30 \mathrm{~Hz}, 24 \mathrm{~dB} /$ octave), and eye movement correction using the procedure described by Gratton, Coles, and Donchin (1983). Stimulus-locked epochs were created from -100 to 
2,000 ms. Artifact in the EEG signal was identified if an amplitude was larger than $\pm 100 \mu \mathrm{V}$ in the epoch. Given that several participants had an insufficient number of artifact-free incorrect source trials, the ERP analysis only focused on correct source and correct rejection trials. The number of trials included in each average did not significantly differ between the exercise and rest conditions (correct source: 61 vs. 72; correct rejection: 53 vs. $57), t \mathrm{~s}(17)<0.81, p \mathrm{~s} \geq .05, d \mathrm{~s} \leq 0.85$. According to previous studies (Curran, 2000; Curran \& Cleary, 2003; Herzmann et al., 2012; Woroch \& Gonsalves, 2010), ERP waveforms were averaged over four electrode sites: left frontal (F3, F5, FC3, and FC5) and right frontal (F4, F6, FC4, and FC6) areas for the FN400 old/new effect, and left parietal (CP3, CP5, P3, and P5) and right parietal (CP4, CP6, P4, and P6) areas for the parietal old/ new effect. Based on previous findings (Curran, 2000; Herzmann et al., 2012; Wilding \& Rugg, 1996; Woroch \& Gonsalves, 2010) and visual inspection of the grand average waveforms, the mean amplitudes were calculated in two time windows, from 200 to $400 \mathrm{~ms}$ (FN400 old/new effect) and from 400 to $900 \mathrm{~ms}$ (parietal old/new effect) after stimulus onset.

\section{Statistical Analysis}

Inverse sine transformation was applied to all percentage data. For the encoding phase, response accuracy and RT were analyzed using a 2 (Condition: exercise, rest) $\times 2$ (Block: size, animacy) repeated-measures analysis of variance. For the retrieval phase, hit, correct source, and correct rejection accuracy were analyzed using paired $t$ tests between conditions. RTs on hit, miss, and correct rejection trials were analyzed using paired $t$ tests between conditions. Mean amplitudes of ERP components were analyzed using a 2 (Condition: exercise, rest) $\times 2$ (Trial: correct source, correct rejection) $\times 2$ (Site: left, right) repeated-measures analysis of variance. Analyses with three within-participants levels employed the Greenhouse-Geisser statistic if the sphericity assumption was violated. Post hoc analyses were conducted using Bonferroni-corrected $t$ tests. Effect sizes are presented as partial eta-squared $\left(\eta_{\mathrm{p}}^{2}\right)$ and Cohen's $d$ for analysis of variances and $t$ tests, respectively. The significant threshold of all statistical analysis was set at $p=.05$.

\section{Results}

\section{Task Performance}

Encoding phase. Analysis of response accuracy revealed a main effect of Block, $F(1,17)=34.2, p<.001$, $\eta_{\mathrm{p}}^{2}=.67$, with lower accuracy for the size block (mean $=$ $95.1 \%, S D=2.7$ ) relative to the animacy block (mean $=$ $98.0 \%, S D=1.9)$. No main effect or interaction involving the Condition factor was observed, $F \mathrm{~s}(1,17) \leq .23$, $p \mathrm{~s} \geq .64, \eta_{\mathrm{p}}^{2} \mathrm{~s} \leq .01$.

Analysis of RTs revealed a main effect of Block, $F(1,17)=15.9, p=.001, \eta_{p}^{2}=.48$, with longer RT for the size block (mean $=1,156.4 \mathrm{~ms}, S D=319.2)$ relative to the animacy block (mean $=1,006.9 \mathrm{~ms}, S D=294.0)$. No main effect or interaction involving the Condition factor was observed, $F \mathrm{~s}(1,17) \leq .14, p \mathrm{~s} \geq .71, \eta_{\mathrm{p}}^{2} \leq .01$.

Retrieval phase. Figure 2 illustrates mean hit, correct source, and correct rejection accuracy in each condition. Correct source accuracy was lower in the exercise
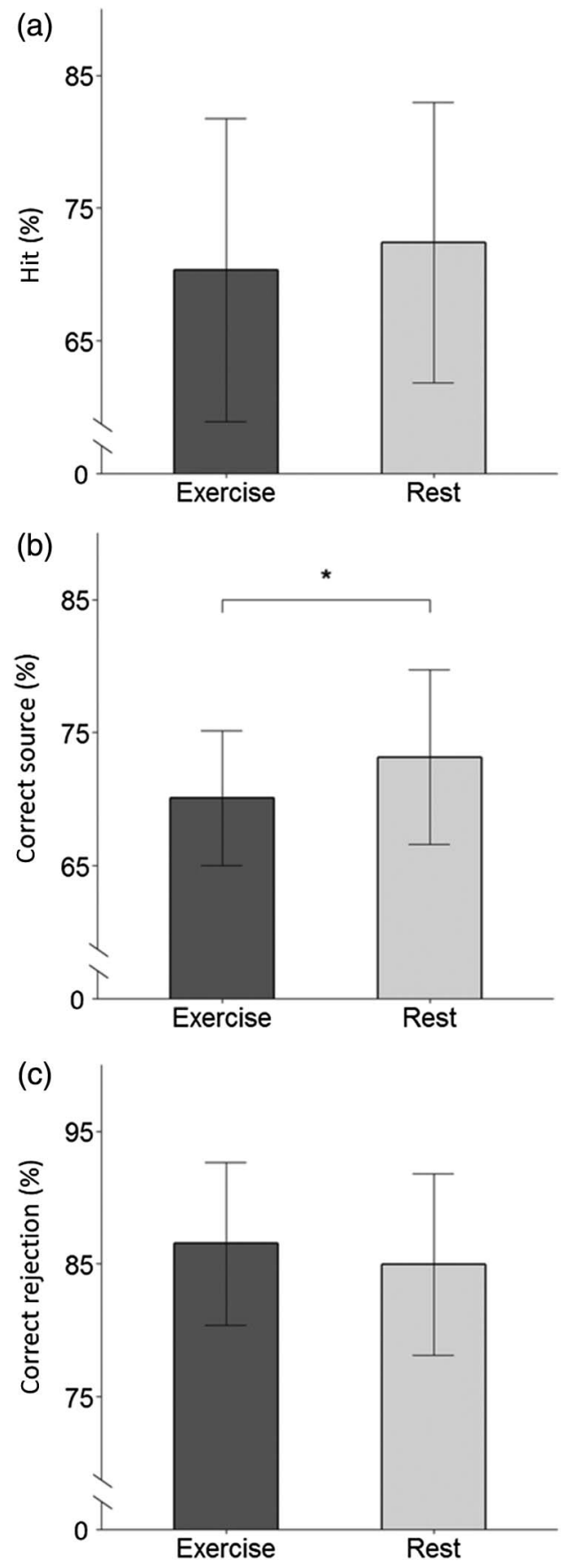

Figure 2 - Mean (SD) of hit (a), correct source (b), and correct rejection (c) accuracy during the retrieval phase in each condition, $* p<.05$. 


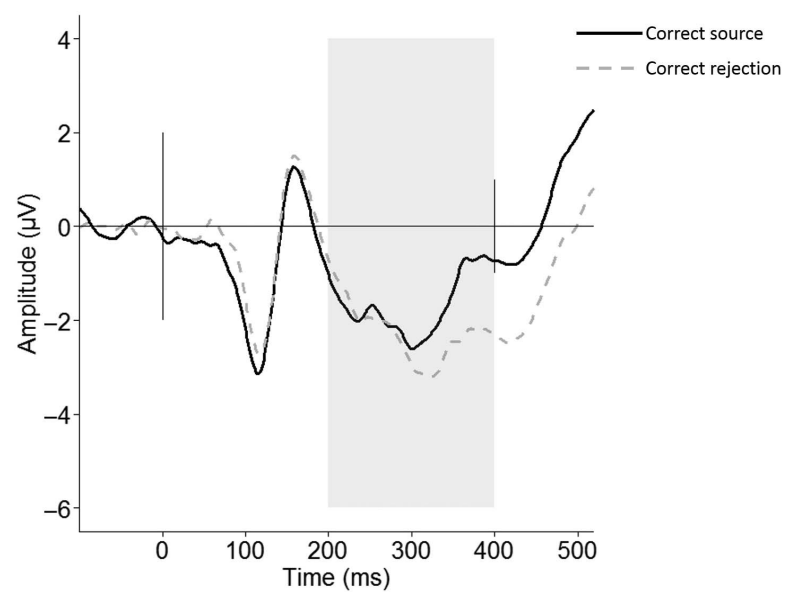

Figure 3 - Grand average ERP waveforms for each trial in the frontal area. Gray shadows show the time window for the FN400 old/new effect. The Condition factor is collapsed in this figure. $\mathrm{ERP}=$ event-related brain potential.

condition relative to the rest condition, $t(17)=2.1$, $p<.05, d=0.54$. No significant difference was observed for hit and correct rejection accuracy, $t \mathrm{~s}(17) \leq 1.2, p \mathrm{~s} \geq$ $.25, d \mathrm{~s} \leq 0.26$. RTs on hit, miss, correct rejection trials did not differ between conditions, $t \mathrm{~s}(17) \leq 0.66, p \mathrm{~s} \geq .52$, $d \mathrm{~s} \leq 0.12$.
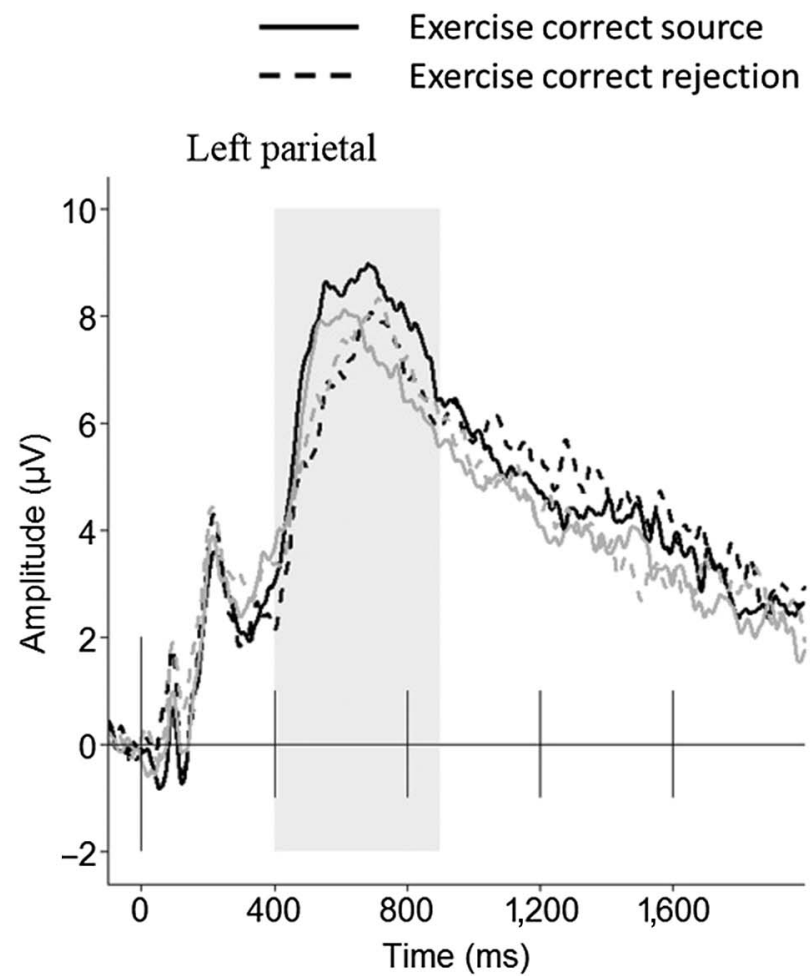

\section{Event-Related Brain Potentials}

FN400 old/new effect. Figure 3 illustrates grand average ERP waveforms at left and right frontal areas (i.e., FN400 old/new effect). Analysis of mean amplitude revealed a main effect of Trial, $F(1,17)=10.0$, $p=.006, \eta_{\mathrm{p}}^{2}=.37$, with more positive amplitude for the correct source trials (mean $=-1.5 \mu \mathrm{V}, S D=3.8$ ) relative to the correct rejection trials (mean $=-2.2 \mu \mathrm{V}, S D=4.0$ ), confirming the FN400 old/new effect. No main effect or interaction involving the Condition factor were observed, $F \mathrm{~s}(1,17) \leq 0.35, p \mathrm{~s} \geq .56, \eta_{\mathrm{p}}^{2} \leq .02$.

Parietal old/new effect. Figure 4 illustrates grand average ERP waveforms for each condition and trial at left and right parietal areas (i.e., parietal old/new effect). Analysis of mean amplitude revealed an interaction of Trial $\times$ Site, $F(1,17)=5.6, p=.03, \eta_{\mathrm{p}}^{2}=.25$, which was superseded by an interaction of Condition $\times$ Trial $\times$ Site, $F(1,17)=11.0, p<.01, \eta_{\mathrm{p}}^{2}=.39$. Figure 5 illustrates the Condition $\times$ Trial $\times$ Site interaction. Decomposition of the three-way interaction examined Trial $\times$ Site within each condition and revealed a Trial $\times$ Site interaction in the exercise condition, $F(1,17)=17.4, p<.01, \eta_{\mathrm{p}}^{2}=.51$, but not in the rest condition, $F(1,17)=0.09, p=.76$, $\eta_{\mathrm{p}}^{2}=.01$. Post hoc analyses for the exercise condition revealed larger amplitude for the correct source relative to the correct rejection trials at the left parietal area, $t(17)=2.2, p=.04, d=0.31$, confirming the parietal

\section{- Rest correct source \\ - - - Rest correct rejection}

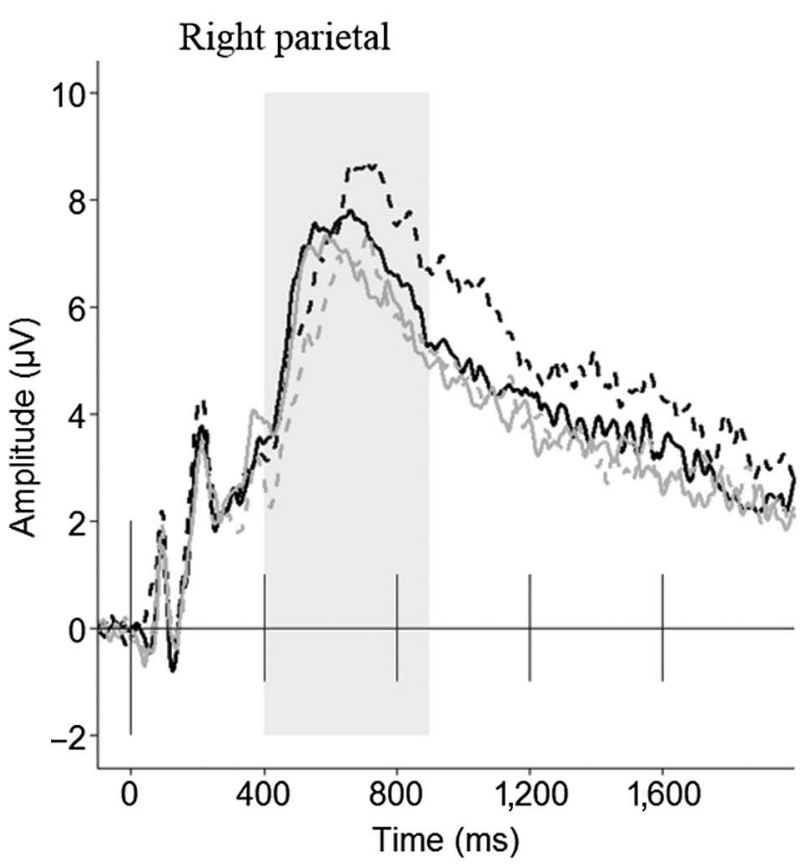

Figure 4 - Grand average ERP waveforms for each condition and trial at left and right parietal areas. Gray shadows show the time window for the parietal old/new effect. ERP = event-related brain potential. 


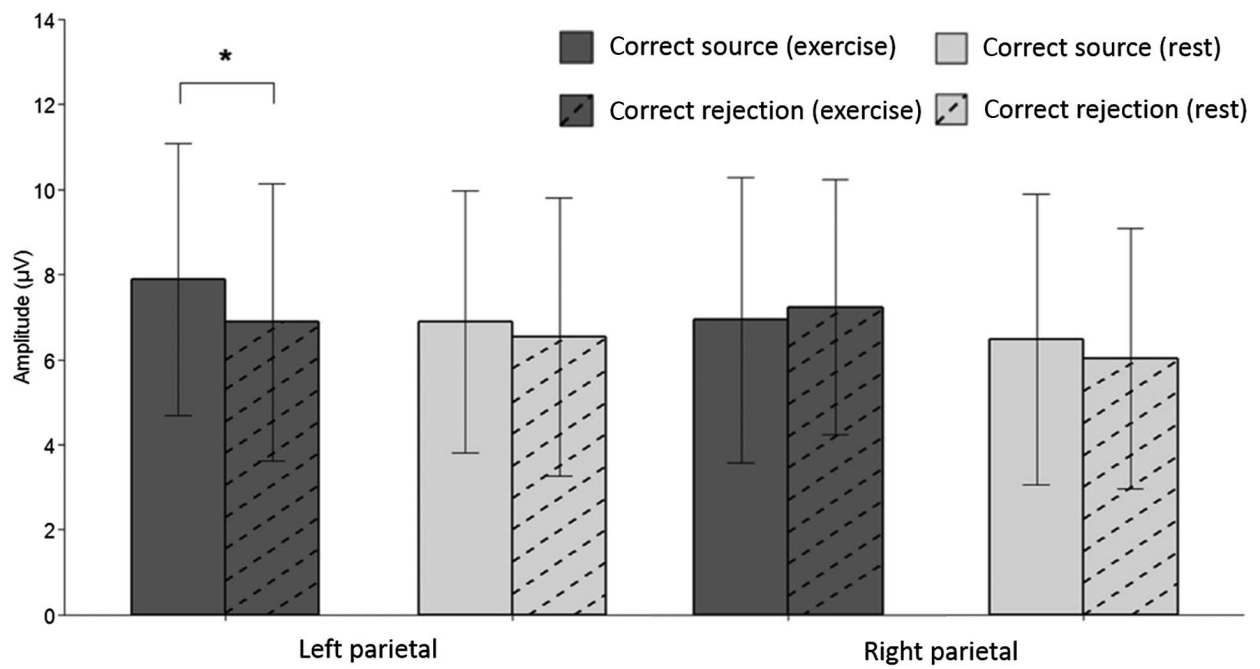

Figure 5 - Mean $(S D)$ parietal amplitude for each condition, trial, and site illustrating a Condition $\times$ Trial $\times$ Site interaction, $* p<.05$.

old/new effect, whereas no such difference was observed at the right parietal area, $t(17)=0.70, p=.49, d=0.09$. In the rest condition, the amplitude did not differ between trials, $F(1,17)=0.93, p=.35, \eta_{\mathrm{p}}^{2}=.05$, indicating absence of the parietal old/new effect.

\section{Discussion}

The aim of this study was to clarify the effects of aerobic exercise during encoding on hippocampus-dependent memory function by using a source memory task. Consistent with our hypothesis, correct source accuracy was lower in the exercise condition, relative to the rest condition, whereas old/new accuracy (i.e., hit and correct rejection accuracy) did not differ between the conditions. These findings suggest that aerobic exercise during encoding impaired the recollection processing, whereas the familiarity process remained unaffected. Neuroimaging studies have shown that the hippocampus selectively supports the recollection process, whereas adjacent cortex in the medial temporal lobe supports the familiarity process (Cansino et al., 2002; Hoppstadter et al., 2015; Yonelinas et al., 2005). Thus, the behavioral findings of this study suggest that simultaneous exercise and encoding selectively impairs hippocampal functioning.

These findings support prior research suggesting the performance of executive function tasks, such as the flanker and $n$-back tasks, decreases during aerobic exercise (Pontifex \& Hillman, 2007; Soga et al., 2015). It is well established that executive function is primarily mediated by the DLPFC (Alvarez \& Emory, 2006; Funahashi, 2001; Smith \& Jonides, 1999). The DLPFC is also likely to assume a critical role in encoding source information (Murray \& Ranganath, 2007). Further, according to the transient hypofrontality hypothesis (Dietrich, 2003; Dietrich \& Audiffren, 2011), during exercise, neural activity temporarily decreases in brain areas such as the prefrontal cortex that are not directly involved in the exercise task. Taken together, it is reasonable to assume that reduced correct source accuracy in the exercise condition is due to decreased functioning of both the hippocampal and DLPFC, and/or a prefrontal-hippocampal interactions during aerobic exercise.

The negative effects of aerobic exercise observed in this study are consistent with the findings of Miles and Hardman (1998). They focused on state-dependent learning and reported that free recall performance was better in the same-state encoding condition (i.e., rest-rest and exercise-exercise for encoding-retrieval) than in the changed-state encoding condition (i.e., exercise-rest and rest-exercise for encoding-retrieval). In this study, the retrieval phase was performed during seated rest in both conditions, such that exercise and rest conditions corresponded to the changed-state and same-state encoding conditions, respectively. Thus, the state-dependent learning explanation is one possible interpretation of the negative effects observed in this study.

However, behavioral findings of the current study contradict those of Schmidt-Kassow et al. (2013, 2014), who suggested that aerobic exercise during encoding improved memory function. Several methodological differences between the current and the previous study might explain these inconsistent results. First, SchmidtKassow et al. used a vocabulary learning task, in which native German participants learned Polish vocabulary. As discussed previously, the present study demonstrated that aerobic exercise during encoding selectively decreased recollection. It is possible that the current source memory task made greater recollection demands than the vocabulary learning task. Thus, the effects of acute aerobic exercise might differ depending on the nature of the memory task. Second, Schmidt-Kassow et al. (2013) conducted the same two experimental 
sessions to examine whether familiarization with the dual-task situation (i.e., exercise and encoding) influenced the effects of doing aerobic exercise during encoding on memory function. They indicated that aerobic exercise during encoding improved vocabulary learning, and this positive effect was greater for the second session. These findings suggest that familiarization with the dual-task situation might improve retrieval performance of participants that conducted a memory-encoding task during exercise. Thus, although the results of this study indicated that acute exercise during encoding had a negative effect on hippocampus-dependent memory functions, these negative effects might be attenuated, or even reversed, if participants become familiar with the dual-task situation.

As hypothesized, ERP data indicated that the effects of acute aerobic exercise were selectively observed for the parietal old/new effect, but not for the FN400 old/ new effect. These ERP findings are partially consistent with task performance data, suggesting that aerobic exercise during encoding selectively influenced recollection, but not familiarity processing. As stated in the beginning of this article, we predicted that the negative effects of acute aerobic exercise would be reflected in a decreased parietal old/new effect. However, our results indicated that the parietal old/new effect was found in the exercise condition but absent in the rest condition, which was the opposite of our prediction. Hoppstadter et al. (2015) examined brain activations that were associated with the parietal old/new effect using simultaneous EEG-functional magnetic resonance imaging recordings and found a relationship between hippocampal activation and the parietal old/new effect. Therefore, it is likely that the observed parietal old/new effect in the exercise condition were indicative of exercise-induced hippocampal activation. Despite the post-acute exercise effect however, correct source accuracy was lower in the exercise, relative to the rest condition. One possible explanation for these contradictory results is that participants had more trouble encoding contextual information during exercise than seated rest. Although speculative, it is concluded that lower source accuracy might have been observed in the exercise condition due to inefficient source encoding during aerobic exercise.

\section{Limitations}

Several limitations of this study should be noted. First, results suggested that lower correct accuracy observed in the exercise condition might be attributed to deterioration in encoding efficiency during aerobic exercise. However, the results are limited because ERP data during the encoding phase were not analyzed due to excessive noise, which is a major weakness of this study. Recent research has facilitated measuring EEG data during exercise (Gramann, Gwin, Bigdely-Shamlo, Ferris, \& Makeig, 2010; Gwin, Gramann, Makeig, \& Ferris, 2010, 2011). This newly developed methodology for analyzing EEG during exercise could provide fresh insights into the effect of acute aerobic exercise on hippocampus-dependent memory function.

Second, given that the retrieval phase was performed $5 \mathrm{~min}$ after the encoding phase, a postacute exercise effect was included in the exercise condition, but not in the rest condition. As discussed previously, however, behavioral and ERP data indicated that correct source accuracy was lower in the exercise condition even though hippocampal activity increased after aerobic exercise, which is suggestive of inefficient source encoding during aerobic exercise. Therefore, it is possible that the postacute exercise effect did not have a critical impact on the present results. It is suggested that the retrieval phase should be conducted several hours after exercise to exclude the post-acute exercise effect in future studies, similar to the studies by Schmidt-Kassow et al. $(2013,2014)$.

Third, negative effects of aerobic exercise that were observed in this study might merely reflect the effects of state-dependent learning and not the effect of acute aerobic exercise per se. In the exercise condition, the encoding and the retrieval phases were performed, respectively, on a cycle ergometer and a chair, whereas in the rest condition, both phases were conducted on a chair. If participants had performed the encoding phase on a cycle ergometer without exercising and then performed the retrieval phase on a chair, their performance might have deteriorated compared with the rest condition in the current study. Schmidt-Kassow et al. (2013) found that cycling exercise during encoding improved vocabulary learning even though the retrieval phase was not performed on a cycle ergometer. This finding suggesting that minor state differences, such as sitting on a cycle ergometer versus a chair, are unrelated to statedependent learning is inconsistent with the theory of state-dependent learning. Therefore, it is plausible that the observed deterioration of retrieval performance in the exercise condition was due to simultaneous exercising and encoding. Nevertheless, it is suggested that a more rigorously controlled study is needed to clarify the influence of state-dependent learning.

Last, the parietal old/new effect was absent in the rest condition, which is inconsistent with previous findings (Addante et al., 2012; Cansino \& Trejo-Morales, 2008; Curran, 2000; Herzmann et al., 2012; Wilding \& Rugg, 1996). Methodological differences, such as stimuli (e.g., picture or word) and source judgments (i.e., old/new and source judgments were performed separately or simultaneously), might be responsible for this discrepancy. Resolving this issue is beyond the scope of this study. More importantly, the parietal old/new effect was found in the exercise condition, which is suggestive of exercise-induced hippocampal activation.

\section{Conclusion}

We used behavioral and neuroelectric indices of recollection and demonstrated that hippocampus-dependent memory function is negatively affected by conducting 
aerobic exercise during encoding. Moreover, aerobic exercise did not necessarily have any beneficial effects on hippocampus-dependent memory function. Therefore, caution is suggested in encouraging physically active academic lessons, in which exercises are simultaneously conducted with learning activities. It is hoped that these findings will contribute to a deeper understanding of the effects of acute exercises on brain functions.

\section{Acknowledgments}

This study was supported by a grant from Ozaki Co., Ltd., and JSPS KAKENHI grant numbers $17 \mathrm{H} 02139$ from the Japan Society for the Promotion of Science and MEXT-Supported Program for the Strategic Research Foundation at Private Universities, 2015-2019 from the Ministry of Education, Culture, Sports, Science and Technology (S1511017).

\section{Note}

1. Studies comparing correct and incorrect source trials have consistently shown a more positive amplitude for the correct source, relative to incorrect source trials in the parietal old/ new effect time window supporting the notion that the parietal old/new effect is associated with recollection processing. Moreover, findings on the FN400 old/new effect time window have been inconsistent, with certain studies indicating a comparable amplitude between correct source and incorrect source trials (Mollison \& Curran, 2012), and other studies showing a more positive amplitude for correct source relative to incorrect source trials (Addante et al., 2012; Cansino \& Trejo-Morales, 2008; Peters \& Daum, 2009). This difference could be the result of differences in the source information, such as location or color (Mollison \& Curran, 2012). The latter effect might reflect the contribution of the degree of familiarity on source recognition (Addante et al., 2012). Please note that incorrect source trials could not be analyzed in this study because of the insufficient number of trials to compute ERP averages.

\section{References}

Addante, R.J., Ranganath, C., \& Yonelinas, A.P. (2012). Examining ERP correlates of recognition memory: Evidence of accurate source recognition without recollection. Neuroimage, 62(1), 439-450. PubMed doi:10.1016/j. neuroimage.2012.04.031

Alvarez, J.A., \& Emory, E. (2006). Executive function and the frontal lobes: A meta-analytic review. Neuropsychology Review, 16(1), 17-42. PubMed doi:10.1007/s11065-0069002-x

Bonin, P., Peereman, R., Malardier, N., Meot, A., \& Chalard, M. (2003). A new set of 299 pictures for psycholinguistic studies: French norms for name agreement, image agreement, conceptual familiarity, visual complexity, image variability, age of acquisition, and naming latencies.
Behavior Research Methods, Instruments, \& Computers, 35(1), 158-167. PubMed doi:10.3758/BF03195507

Bull, F.C., Maslin, T.S., \& Armstrong, T. (2009). Global physical activity questionnaire (GPAQ): Nine country reliability and validity study. Journal of Physical Activity \& Health, 6(6), 790-804. PubMed doi:10.1123/jpah.6. 6.790

Cabeza, R., Ciaramelli, E., Olson, I.R., \& Moscovitch, M. (2008). The parietal cortex and episodic memory: An attentional account. Nature Reviews Neuroscience, 9(8), 613-625. PubMed doi:10.1038/nrn2459

Cansino, S., Maquet, P., Dolan, R.J., \& Rugg, M.D. (2002). Brain activity underlying encoding and retrieval of source memory. Cerebral Cortex, 12(10), 1048-1056. PubMed doi:10.1093/cercor/12.10.1048

Cansino, S., \& Trejo-Morales, P. (2008). Neurophysiology of successful encoding and retrieval of source memory. Cognitive, Affective \& Behavioral Neuroscience, 8(1), 85-98. PubMed doi:10.3758/CABN.8.1.85

Chaddock, L., Erickson, K.I., Prakash, R.S., Kim, J.S., Voss, M.W., Vanpatter, M., ... Kramer, A.F. (2010). A neuroimaging investigation of the association between aerobic fitness, hippocampal volume, and memory performance in preadolescent children. Brain Research, 1358, 172-183. PubMed doi:10.1016/j.brainres.2010.08.049

Chaddock, L., Hillman, C.H., Buck, S.M., \& Cohen, N.J. (2011). Aerobic fitness and executive control of relational memory in preadolescent children. Medicine \& Science in Sports \& Exercise, 43(2), 344-349. PubMed doi:10. 1249/MSS.0b013e3181e9af48

Chang, Y.K., Labban, J.D., Gapin, J.I., \& Etnier, J.L. (2012). The effects of acute exercise on cognitive performance: A meta-analysis. Brain Research, 1453, 87-101. PubMed doi:10.1016/j.brainres.2012.02.068

Chatrian, G.E., Lettich, E., \& Nelson, P.L. (1985). Ten percent electrode system for topographic studies of spontaneous and evoked EEG activities. American Journal of EEG Technology, 25(2), 83-92. doi:10.1080/00029238.1985. 11080163

Curran, T. (2000). Brain potentials of recollection and familiarity. Memory \& Cognition, 28(6), 923-938. PubMed doi:10.3758/BF03209340

Curran, T., \& Cleary, A.M. (2003). Using ERPs to dissociate recollection from familiarity in picture recognition. Cognitive Brain Research, 15(2), 191-205. PubMed doi:10. 1016/S0926-6410(02)00192-1

Curran, T., \& Hancock, J. (2007). The FN400 indexes familiarity-based recognition of faces. NeuroImage, 36(2), 464-471. PubMed doi:10.1016/j.neuroimage.2006. 12.016

Cycowicz, Y.M., Friedman, D., Rothstein, M., \& Snodgrass, J.G. (1997). Picture naming by young children: Norms for name agreement, familiarity, and visual complexity. Journal of Experimental Child Psychology, 65(2), 171-237. PubMed doi:10.1006/jecp.1996.2356

Dietrich, A. (2003). Functional neuroanatomy of altered states of consciousness: The transient hypofrontality hypothesis. Consciousness and Cognition, 12(2), 231-256. PubMed doi:10.1016/S1053-8100(02)00046-6 
Dietrich, A, \& Audiffren, M. (2011). The reticular-activating hypofrontality (RAH) model of acute exercise. Neuroscience \& Biobehavioral Reviews, 35(6), 1305-1325. PubMed doi:10.1016/j.neubiorev.2011.02.001

Dietrich, A., \& Sparling, P.B. (2004). Endurance exercise selectively impairs prefrontal-dependent cognition. Brain and Cognition, 55(3), 516-524. PubMed doi:10.1016/ j.bandc.2004.03.002

Dolcos, F., LaBar, K.S., \& Cabeza, R. (2005). Remembering one year later: Role of the amygdala and the medial temporal lobe memory system in retrieving emotional memories. Proceedings of the National Academy of Sciences of the United States of America, 102(7), 2626-2631. PubMed doi:10.1073/pnas.0409848102

Donnelly, J.E., \& Lambourne, K. (2011). Classroom-based physical activity, cognition, and academic achievement. Preventive Medicine, 52(Suppl. 1), S36-S42. PubMed doi:10.1016/j.ypmed.2011.01.021

Eichenbaum, H., Yonelinas, A.P., \& Ranganath, C. (2007). The medial temporal lobe and recognition memory. Annual Review of Neuroscience, 30, 123-152. PubMed doi:10.1146/annurev.neuro.30.051606.094328

Erickson, K.I., Hillman, C.H., \& Kramer, A.F. (2015). Physical activity, brain, and cognition. Current Opinion in Behavioral Sciences, 4, 27-32. doi:10.1016/j.cobeha.2015. 01.005

Erickson, K.I., Prakash, R.S., Voss, M.W., Chaddock, L., Hu, L., Morris, K.S., . . Kramer, A.F. (2009). Aerobic fitness is associated with hippocampal volume in elderly humans. Hippocampus, 19(10), 1030-1039. PubMed doi:10. 1002/hipo. 20547

Erickson, K.I., Voss, M.W., Prakash, R.S., Basak, C., Szabo, A., Chaddock, L., ... Kramer, A.F. (2011). Exercise training increases size of hippocampus and improves memory. Proceedings of the National Academy of Sciences of the United States of America, 108(7), 3017-3022. PubMed doi:10.1073/pnas.1015950108

Etnier, J., Labban, J.D., Piepmeier, A., Davis, M.E., \& Henning, D.A. (2014). Effects of an acute bout of exercise on memory in 6th grade children. Pediatric Exercise Science, 26(3), 250-258. PubMed doi:10.1123/pes. 2013-0141

Funahashi, S. (2001). Neuronal mechanisms of executive control by the prefrontal cortex. Neuroscience Research, 39(2), 147-165. PubMed doi:10.1016/S0168-0102(00) 00224-8

Gramann, K., Gwin, J.T., Bigdely-Shamlo, N., Ferris, D.P., \& Makeig, S. (2010). Visual evoked responses during standing and walking. Frontiers in Human Neuroscience, 4, 202. PubMed doi:10.3389/fnhum.2010.00202

Gratton, G., Coles, M.G., \& Donchin, E. (1983). A new method for off-line removal of ocular artifact. Electroencephalography \& Clinical Neurophysiology, 55(4), 468484. PubMed doi:10.1016/0013-4694(83)90135-9

Gwin, J.T., Gramann, K., Makeig, S., \& Ferris, D.P. (2010). Removal of movement artifact from high-density EEG recorded during walking and running. Journal of Neurophysiology, 103(6), 3526-3534. PubMed doi:10.1152/ jn.00105.2010
Gwin, J.T., Gramann, K., Makeig, S., \& Ferris, D.P. (2011). Electrocortical activity is coupled to gait cycle phase during treadmill walking. Neuroimage, 54(2), 12891296. PubMed doi:10.1016/j.neuroimage.2010.08.066

Herzmann, G., Jin, M., Cordes, D., \& Curran, T. (2012). A within-subject ERP and fMRI investigation of orientation-specific recognition memory for pictures. Cognitive Neuroscience, 3(3-4), 174-192. doi:10.1080/17588928. 2012.669364

Hillman, C.H., Pontifex, M.B., Raine, L.B., Castelli, D.M., Hall, E.E., \& Kramer, A.F. (2009). The effect of acute treadmill walking on cognitive control and academic achievement in preadolescent children. Neuroscience, 159(3), 1044-1054. PubMed doi:10.1016/j.neuroscience. 2009.01.057

Hoppstadter, M., Baeuchl, C., Diener, C., Flor, H., \& Meyer, P. (2015). Simultaneous EEG-fMRI reveals brain networks underlying recognition memory ERP old/new effects. NeuroImage, 116, 112-122. PubMed doi:10.1016/ j.neuroimage.2015.05.026

Konkel, A., \& Cohen, N.J. (2009). Relational memory and the hippocampus: Representations and methods. Frontiers in Neuroscience, 3(2), 166-174. PubMed doi:10.3389/ neuro.01.023.2009

Labban, J.D., \& Etnier, J.L. (2011). Effects of acute exercise on long-term memory. Research Quarterly for Exercise and Sport, 82(4), 712-721. PubMed doi:10.1080/02701367. 2011.10599808

Lambourne, K., \& Tomporowski, P. (2010). The effect of exercise-induced arousal on cognitive task performance: A meta-regression analysis. Brain Research, 1341, 12-24. PubMed doi:10.1016/j.brainres.2010.03.091

Miles, C., \& Hardman, E. (1998). State-dependent memory produced by aerobic exercise. Ergonomics, 41(1), 20-28. PubMed doi:10.1080/001401398187297

Mollison, M.V., \& Curran, T. (2012). Familiarity in source memory. Neuropsychologia, 50(11), 2546-2565. PubMed doi:10.1016/j.neuropsychologia.2012.06.027

Mullender-Wijnsma, M.J., Hartman, E., de Greeff, J.W., Bosker, R.J., Doolaard, S., \& Visscher, C. (2015). Improving academic performance of school-age children by physical activity in the classroom: 1-year program evaluation. Journal of School Health, 85(6), 365-371. PubMed doi:10.1111/josh.12259

Murray, L.J., \& Ranganath, C. (2007). The dorsolateral prefrontal cortex contributes to successful relational memory encoding. The Journal of Neuroscience, 27(20), 5515-5522. PubMed doi:10.1523/JNEUROSCI.040607.2007

Nishimoto, T., Ueda, T., Miyawaki, K., Une, Y., \& Takahashi, M. (2012). The role of imagery-related properties in picture naming: A newly standardized set of 360 pictures for Japanese. Behavior Research Methods, 44(4), 934945. PubMed doi:10.3758/s13428-011-0176-7

Pesce, C, Crova, C, Cereatti, L, Casella, R, \& Bellucci, M. (2009). Physical activity and mental performance in preadolescents: Effects of acute exercise on free-recall memory. Mental Health and Physical Activity, 2(1), 16-22. doi:10.1016/j.mhpa.2009.02.001 
Peters, J., \& Daum, I. (2009). Frontal but not parietal positivity during source recollection is sensitive to episodic content. Neuroscience Letters, 454(3), 182-186. doi:10.1016/ j.neulet.2009.03.019

Pontifex, M.B., \& Hillman, C.H. (2007). Neuroelectric and behavioral indices of interference control during acute cycling. Clinical Neurophysiology, 118(3), 570-580. PubMed doi:10.1016/j.clinph.2006.09.029

Pontifex, M.B., Hillman, C.H., Fernhall, B., Thompson, K.M., $\&$ Valentini, T.A. (2009). The effect of acute aerobic and resistance exercise on working memory. Medicine \& Science in Sports \& Exercise, 41(4), 927-934. PubMed doi:10.1249/MSS.0b013e3181907d69

Roig, M., Nordbrandt, S., Geertsen, S.S., \& Nielsen, J.B. (2013). The effects of cardiovascular exercise on human memory: A review with meta-analysis. Neuroscience \& Biobehavioral Reviews, 37(8), 1645-1666. PubMed doi:10.1016/j.neubiorev.2013.06.012

Salas, C.R., Minakata, K., \& Kelemen, W.L. (2011). Walking before study enhances free recall but not judgementof-learning magnitude. Journal of Cognitive Psychology, 23(4), 507-513. doi:10.1080/20445911.2011.532207

Schmidt-Kassow, M., Deusser, M., Thiel, C., Otterbein, S., Montag, C., Reuter, M., ... Kaiser, J. (2013). Physical exercise during encoding improves vocabulary learning in young female adults: A neuroendocrinological study. PLoS ONE, 8(5), e64172. PubMed doi:10.1371/journal. pone. 0064172

Schmidt-Kassow, M., Zink, N., Mock, J., Thiel, C., Vogt, L., Abel, C., \& Kaiser, J. (2014). Treadmill walking during vocabulary encoding improves verbal long-term memory. Behavioral and Brain Functions, 10, 24. PubMed doi:10. 1186/1744-9081-10-24

Senkfor, A.J., \& Van Petten, C. (1998). Who said what? An event-related potential investigation of source and item memory. Journal of Experimental Psychology: Learning, Memory, and Cognition, 24(4), 1005-1025. PubMed

Smith, E.E., \& Jonides, J. (1999). Storage and executive processes in the frontal lobes. Science, 283(5408), 1657-1661. PubMed doi:10.1126/science.283.5408.1657

Soga, K, Shishido, T, \& Nagatomi, R. (2015). Executive function during and after acute moderate aerobic exercise in adolescents. Psychology of Sport and Exercise, 16, 7-17. doi:10.1016/j.psychsport.2014.08.010
Tanaka, H., Monahan, K.D., \& Seals, D.R. (2001). Agepredicted maximal heart rate revisited. Journal of the American College of Cardiology, 37(1), 153-156. PubMed doi:10.1016/S0735-1097(00)01054-8

Thomas, S., Reading, J., \& Shephard, R.J. (1992). Revision of the Physical Activity Readiness Questionnaire (PAR-Q). Canadian Journal of Sport Sciences, 17(4), 338-345. PubMed

Tsivilis, D., Allan, K., Roberts, J., Williams, N., Downes, J.J., \& El-Deredy, W. (2015). Old-new ERP effects and remote memories: The late parietal effect is absent as recollection fails whereas the early mid-frontal effect persists as familiarity is retained. Frontiers in Human Neuroscience, 9, 532. doi:10.3389/fnhum.2015.00532

Weng, T.B., Pierce, G.L., Darling, W.G., \& Voss, M.W. (2015). Differential effects of acute exercise on distinct aspects of executive function. Medicine \& Science in Sports \& Exercise, 47(7), 1460-1469. PubMed doi:10. 1249/MSS.0000000000000542

Wilding, E.L. (2000). In what way does the parietal ERP old/ new effect index recollection? International Journal of Psychophysiology, 35(1), 81-87. PubMed doi:10.1016/ S0167-8760(99)00095-1

Wilding, E.L., \& Rugg, M.D. (1996). An event-related potential study of recognition memory with and without retrieval of source. Brain, 119(Pt. 3), 889-905. PubMed doi:10.1093/brain/119.3.889

Winter, B., Breitenstein, C., Mooren, F.C., Voelker, K., Fobker, M., Lechtermann, A., ... Knecht, S. (2007). High impact running improves learning. Neurobiology of Learning and Memory, 87(4), 597-609. PubMed doi:10. 1016/j.nlm.2006.11.003

Woroch, B., \& Gonsalves, B.D. (2010). Event-related potential correlates of item and source memory strength. Brain Research, 1317, 180-191. PubMed doi:10.1016/j. brainres.2009.12.074

Yonelinas, A.P., Aly, M., Wang, W.C., \& Koen, J.D. (2010). Recollection and familiarity: Examining controversial assumptions and new directions. Hippocampus, 20(11), 1178-1194. PubMed doi:10.1002/hipo.20864

Yonelinas, A.P., Otten, L.J., Shaw, K.N., \& Rugg, M.D. (2005). Separating the brain regions involved in recollection and familiarity in recognition memory. The Journal of Neuroscience, 25(11), 3002-3008. PubMed doi:10. 1523/JNEUROSCI.5295-04.2005 